\title{
BIBLIOGRAFIA
}

\section{Continuación de una Bibliografía de y sobre Juan Rulfo}

Esta bibliografía es un suplemento a y continuación de la bibliografía sobre la obra de Juan Rulfo, preparada por Arthur Ramírez, que apareció en el número de enero-marzo de 1974 de la Revista Iberoamericana (Pp. 135-171). Para facilitar su uso, se presenta en seis divisiones, siguiendo básicamente las categorías de Ramírez:

I. Obras de Juan Rulfo.

II. Materia bibliográfica.

III. Entrevistas y materia de interés biográfico.

IV. Referencias en general a la obra de Rulfo.

V. Reseñas y artículos sobre El llano en llamas o sus cuentos.

VI. Reseñas y artículos sobre Pedro Páramo.

Para mejor integrar la presente lista con la anterior, de vez en cuando se dan referencias, designadas con los números correspondientes, a ciertas inscripciones del trabajo de Ramírez. Otras veces se dan referencias a números correspondientes a varias inscripciones del presente trabajo. En ambos casos se las dan para llamar la atención sobre alguna relación entre las fichas de que se tratan.

E. KENT LIORET

University of North Carolina at Chapel Hill

I. Obras de Juan Rulfo:

A. Traducciones de cuentos, apareciendo en revistas [véase Ramírez, sección I, parte F]. 
1. Rulfo, Juan. "Anacleto Morones." Traducción al francés de Roger Lescot. La Nouvelle Nouvelle Revue Française, IV, 67 (1 juillet de 1958), 76-92.

B. Antología de cuentos [véase Ramírez, sección I, parte G.].

Con cuentos en español

2. Rulfo, Juan. "La cuesta de las comadres", pp. 552-562. En The Literature of Spanish America. A Critical Antbology. IV. 19301967, Angel Flores, ed. New York: Las Américas Publishing Co., 1967.

3. _- "Diles que no me maten!", pp. 191-198. En El cuento bispanoamericano. Antología crítico-bistórica, Seymour Menton, ed. México: Fondo de Cultura Económica, 1964 [véase Ramírez, No. 312 ].

4. - - “. "Diles que no me maten!”, pp. 291-295. En Literatura mexicana (con notas de literatura bispanoamericana y antología). De acuerdo con los programas oficiales, María del Carmen Millán, eda. México: Editorial Esfinge, 1962.

5. - - "Luvina", pp. 65-74. En Narrativa mexicana de boy, Emmanuel Carballo, ed. Madrid: Alianza Editorial (El Libro de Bolsillo, Sección Literatura), 1969.

6. _- . "El llano en llamas", pp. 15-31. En Cuentos bispanoamericanos, Abelardo Gómez Benoit, ed. Lima: Instituto Latinoamericano de Vinculación Cultural, 1964.

7. —. "No oyes ladrar los perros", pp. 758-. En An Antbology of Spanish American Literature, John E. Englekirk, Irving A. Leonard, John T. Reid and John A. Crow, eds., under the auspices: of the Instituto Internacional de Literatura Iberoamericana. 2a. Ed., New York: Appleton, Century, Crofts, 1968.

Con cuentos traducidos

8. —- "Talpa", pp. 32-39. Traducción al inglés de los editores. En New Voices of Hispanic America, Darwin J. Flakoll y Claribel Alegria, eds. Boston: Beacon Press, 1962.

II. Materia bibliográfica:

9. Anon. Pp. 292-293. En La novela bispanoamericana actual. Com- 
pilación de ensayos criticos, Angel Flores y Raúl Silva Cáceres, eds. New York: Las Américas Publishing Co., 1971.

10. - - pp. 165-169. En Recopilación de textos sobre Juan Rulfo. La Habana: Centro de Investigaciones Literarias, Casa de las Américas (Serie Valoración Múltiple), 1969.

11. Ramírez, Arthur. "Hacia una bibliografía de y sobre Juan Rulfo." Revista Iberoamericana, XL, 86 (enero-marzo de 1974), 135-171.

\section{Entrevistas y materia de interés biográfico:}

12. Anon. "El cuento, con mil y un cuentos." Visión (México), (13 de julio de 1974), 45.

13. B. G. C. "Juan Rulfo recibe el Premio Nacional de Letras." Hispania, 52, 2 (May, 1971), 380.

14. Díaz, Janet W. "Premio Biblioteca Breve desierto." Hispania, 57, 1 (March, 1974), 173.

15. Schneider, Luis Mario. "Mexico's Young Writers Tell Why They Write." Américas (ed. en inglés), 14, no. 6 (June, 1962), 28-31.

16. - - "Nueva genetación del cuento mexicano." Américas (ed. en español), 14, no. 7 (julio de 1962), 28-31 [véase No. 15].

17. Sommers, Joseph. "Juan Rulfo. Entrevista." Hispamérica, II, 4-5 (1973), 103-107.

IV. Referencias en general a la obra de Rulfo:

18. Acker, Bertie Wilcox Naylor. "Themes and World View in the Contemporary Mexican Short Story: Rulfo, Arreola and Fuentes." Dissertation Abstracts International, 32 (1972), 5729-A [véase Ramírez, No. 154].

19. Adoum, Jorge Enrique. "El realismo de Ia otra realidad", pp. 204216. En América Latina en su literatura, César Fernández Moreno, coordinador. México: Siglo Veintiuno Editores (Serie América Latina en su cultura), 1972 [Rulfo, pp. 205, 207, 209].

20. Anon. "Litoral" [2a. noticia]. La Gaceta (Fondo de Cultura Económica), Nueva Epoca, no. 31 (julio de 1973), 7.

21. - . "Ten Basic Mexican Novels." Recent Books in Mexico, II, 4 (1956), 1 y 6.

22. Arreola, Juan José (con Emmanuel Carballo), pp. 359-401. En 
Diecinueve protagonistas de la literatura mexicana del siglo veinte. México: Empresa Editoriales, 1965 [Rulfo, pp. 393-394].

23. Aub, Max. "Los orígenes de la novela de la Revolución mexicana", pp. 225-234. En Panorama de la actual literatura latinoamericana. Madrid: Editorial Fundamentos, 1971 [Rulfo, p. 232].

24. —_ "Max Aub ve a tres jalisciences del '18." La Cultura en México (suplemento de Siempre!), no. 284 (26 de julio de 1967),

25. Bárcenas, Angel. (Sobre El arte de Juan Rulfo, de Rodríguez Alcalá.) El Nacional (20 de feb. de 1966), 15.

26. Bareiro Saguier, Rubén. "Encuentro de culturas", pp. 21-40. En América Latina en su literatura, César Fernández Moreno, coordinador. México: Siglo Veintiséis Editores (Serie América Latina en su cultura), 1972 [Rulfo, p. 40].

27. Benedetti, Mario. "Temas y problemas", pp. 354-371. En América Latina en su literatura, César Fernández Moreno, coordinador. México: Siglo Veintiséis Editores (Serie América Latina en su cultura), 1972 [Rulfo, pp. 356, 358, 360, 361, 367].

28. Benet, Juan (con Federico Campbell). "Juan Benet o el azar", pp. 292-310. En Infame turba, de Federico Campbell. Barciona: Editorial Lumen, 1971 [Rulfo, pp. 298, 306].

29. Caballero Bonald, José Manuel (con Federico Campbell). "José Manuel Caballero Bonald o la penetración del satanismo", pp. 312-330. En Infame turba, de Federico Campbell. Barcelona: Editorial Lumen, 1971 [Rulfo, p. 325].

30. Caballero Bonald, José Manuel (con Fernando Tola de Habich y Patricia Grieve). "Sobre la novela española e hispanoamericana. (Opiniones de J. M. Caballero Bonald)." Mapocho (Santiago de Chile), 23 (Primavera, 1970), 39-54.

31. Campbell, Federico. Infame turba. Barcelona: Editorial Lumen, 1971 [Rulfo, pp. 216, 271, 303; véase también Nos. 28, 29 y 96].

32. Cándido, Antonio. "Literatura y subdesarrollo", pp. 335-353. En América Latina en su literatura, César Fernández Moreno, coordinador. México: Siglo Veintiuno Editores (Serie América Latina en su cultura), 1972 [Rulfo, p. 353].

33. Caparroso, Carlos A. "El novelista Juan Rulfo." Nivel, 99 (1971), $1-2$ y 10 .

34. Carballo, Emmanuel. "La actual literatura mexicana", pp. 15-28. En Panorama de la actual literatura altinoamericana. Madrid: Editorial Fundamentos, 1971 [Rulfo, p. 26]. 
35. - "Del costumbrismo al realismo crítico." Espiral (Bogotá), 91 (junio de 1964), 7-32 [véase Ramírez, No. 202].

36. - Diecinueve protagonistas de la literatura mexicana del siglo veinte. México: Empresa Editoriales, 1965 [Rulfo, pp. 79, 361, 431-432, 447; véase también Nos. 22, 115 y 134$].$

37. - . "Los dos caminos de la universalidad en dos cuentistas mexicanos." Humanismo (México), VII, 50-51 (1958), 108-1:0.

38. - Narrativa mexicana de boy. Madrid: Alianza Editorial (El Libro de Bolsillo, Sección Literatura), 1969 [Rulfo, pp. 17-18, 20-21, 22, 26].

39. Castagnino, Raúl H. Escritores hispanoamericanos desde otros ángulos de simpatía. Buenos Aires: Editorial Nova (Biblioteca Arte y Ciencia de la Expresión), 1971.

40. Castellanos, Rosario. La nowela mexicana contemporánea y su valor testimonial. México: Instituto Nacional de la Juventud Mexicana, 1972 [Rulfo, pp. 10-11, 16].

41. Castellet, José María. "La actual literatura latinoamericana vista desde España", pp. 47-61. En Panorama de la actual literatura latinoamericana. Madrid: Editorial Fundamentos, 1971 [Rulfo, p. 54].

42. Couffon, Claude. "La literatura hispanoamericana vista desde Francia", pp. 294-302. En Panorama de la actual literatura latinoamericana. Madrid: Editorial Fundamentos, 1971 [Rulfo, p. 298.

43. —- "Mexique", pp. 315-320. En Les Littératures contemporaines à travers le monde, Jean-Claude Ibert, ed. Par's: Librairie Hachette, 1961 [Rulfo, pp. 319-320].

44. Cruz, Salvador de la. La novela iberoamericana actual. México: Departamento de Divulgación, Secretaría de Educación Pública, 1956 [Rulfo, pp. 22-24].

45. Cypress, Sandra M. "The Dead Narrator in Modern Latin American Prose Fiction: A Study in Point of View." Urbana, Illinois: University of Illinois, 1968. Tesis doctoral.

46. —. "The Dead Narrator in Modern Latin American Prose Fiction: A Study in Point of View." Dissertation Abstracts, 29 (1969), 2253-A.

47. Del Campo, Xorge. "La natrativa joven de México." Studies in Short Fiction, VIII, 1 (Winter, 1971), 180-198.

48. Deveny, John Joseph, Jr. "Narrative Technique in the Short Stories 
of Juan Rulfo." Gainesville, Florida: The University of Florida, 1973. Tesis doctoral.

49. - - "Narrative Technique in the Short Stories of Juan Rulfo." Dissertation Abstracts International, 34 (1974), 6634 A-6635 A.

50. Díaz, Ramón. "Las razones de Juan Rulfo." Revista de Occidente, 31, no. 93 (dic. de 1970), 344-357.

51. Durán, Manuel. "Juan Rulfo, cuentista: la verdad casi sospechosa", pp. 9-50. En Tríptico mexicano. Juan Rulfo. Carlos Fuentes. Salvador Elizondo. México: Secretaría de Educación Pública (Sep Setentas, no. 18), 1973.

52. ㄴ.. "William Faulkner." Universidad de México, XVI, 12 agosto de 1964), 29.

53. Dután, Manuel y Eva Llorens. "Un gran escritor y su exégeta: Juan Rulfo y Hugo Rodríguez Alcalá." Revista Hispánica Mo. derna, XXXII, 3-4 (julio-oct. de 1966), 242-244.

54. Durand, José. "La última literatura hispanoamericana." Cuddernos (París), 53 (1961), 153-158.

55. Ellis, Keith. "La enseñanza de la literatura hispanoamericana, I." Hispania, 54, no. 3 (sept. de 1973), 617-619).

56. Englekirk, John E., ed. An Outline History of Spanish American Literature. 3a. Ed., Under the Auspices of the Instituto International de Literatura Iberoamericana. New York: Appleton, Century, Crofts, 1965 [Rulfo, pp. 172, 173].

57. Englekirk, John E., Irving A. Leonard, John T. Reid and John A. Crow, eds. "Juan Rulfo", p. 758. En An Antbology of Spanish American Literature. 2a. Ed., Under the Auspices of the Instituto Internacional de Literatura Iberoamericana. New York: Appleton, Century, Crofts, 1968.

58. Ferrer Chívite, Manuel. El laberinto mexicano en/de Juan Rulfo. Madrid: Organización Editorial Novaro, 1972.

59. Ferro, Hellén. "New Novel in Mexico: A Look at Juan Rulfo." Américas (ed. en inglés), 16 (Oct., 1964), 40-41 [véase Ramírez, No. 127].

60. Flakoll, Darwin J., and Claribel Alegría. "Juan Rulfo. Talpa", p. 32. En New Voices of Hispanic America. Boston: Beacon Press, 1962.

61. Flores, Angel. "Juan Rulfo", pp. 549-551. En The Literature of Spanish America. A Critical Antbology. IV. 1930-1967. New York: Las Americas Publishing Co., 1967. 
62. Gallagher, D. F. Modern Latin American Literature. Oxford: Oxford University Press, 1973 [Rulfo, pp. 87, 147-148].

63. González, Manuel Pedro. "Una opinión polémica. A postillas a $\mathrm{La}$ región más transparente." Papel Literario (de El Nacional, Caracas), (22 de mayo de 1966), sin pp.

64. Gordon, Donald K. "Juan Rulfo's Elusive Novel: La cordillera." Hispania, 56, 4 (Dec., 1973), 1040-1041.

65. - The Short Stories of Juan Rulfo." Toronto, University of Toronto, 1969. Tesis doctoral.

66. __. "The Short Stories of Juan Rulfo." Dissertation Abstracts International, 31 (1971), 965 A-966 A.

67. Green, Joan Rea. "The Structure of the Narrator in the Contemporary Spanish American Novel." Austin, Texas: University of Texas, 1970. Tesis doctoral.

68. - - "The Structure of the Narrator in the Contemporary Spanish American Novel." Dissertation Abstracts International, 32 (1971), 6056 A-6057 A.

69. Gullón, Germán. "Similitudes ambientales: Rulfo y Valle-Inclán." Sin Nombre (San Juan, P. R.), I, 4 (abril-junio de 1971), 68-76.

70. Gyurko, Lanin A. "Modern Hispanic-American Fiction: Novel of Action and Narrative of Consciousness." Symposium, XXV, 4 (Winter, 1971), 359-376.

71. Hutchins, J. K. "Literary Life South of the Rio Grande." Saturday Review (Feb. 25, 1967), 42-44.

72. J. F. "Un libro de Méjico. Carta al autor, Juan Rulfo." Indice de Artes y Letras, XI, 99 (1967), 21

73. Jitrik, Noe. "Destrucción y formas en las narraciones", pp. 219242. En América Latina en su literatura, César Fernández Moreno, coordinador. México: Siglo Veintiuno Editores, 1972 [Rulfo, pp. 232, 233, 234-235].

74. Jozef, Bella. História da literatura bispano-americana. Petrópolis, Brasil: Editora Vozes, en Convenio com o Instituto Nacional do Livro, 1971 [Rulfo, pp. 275-277, 305, 307, 347].

75. Kuteistschikova, Vera. "Bemerkungen zur lateinamerikanischen Prosa der Gegenwart." Traducción al alemán del ruso de Gerhard Semekow. Kunst und Literatur (Berlín), 20 (1972), 955-975.

76. —_. "Kontinent, gde vstrechajuts ja vse epokhi: Zametki o sovremennoj latino-amerikanskoj proze." Voprosy Literatury (Moscú), XVI, 4 (1972), 74-97.

77. ㄴ. Meksikanskij Roman. Formirovanie. Svoeobrazie. Sovremen- 
nij Etap. Moscú: Nauka 1971 [Rulfo, 23, 24, 260-267, 288, 307, $309,315]$.

78. Lafforgue, Jorge. "La nueva novela latinoamericana", pp. 13-29. En. Nueva novela latinoamericand $I$, Jorge Lafforgue, compilador. Buenos Aires: Editorial Paidós, 1969.

79. Langford, Walter M. The Mexican Novel Comes of Age. Notre Dame, Indiana: University of Notre Dame Press, 1971 [Rulfo, pp. 38, 73, 85, 88-101, 133, 140, 147, 168, 181, 193, 197, 202, $210,213,217]$.

80, Lescot, Roger. "Juan Rulfo." La Nouvelle Nouvelle Revue Française, VI, 67 (1 juillet de 1958), 159.

81. Luraschi, Ilse Adriana. "Algunos recursos estilisticos en la obra de Juan Rulfo." Pittsburgh: University of Pittsburgh, 1972. Tesis doctoral.

82. - "Algunos recursos estilísticos en la obra de Juan Rulfo." Dissertation Abstracts International, 33 (1973), 6919 A-6920 A.

83. Lyon, Thomas E. "Orderly Observation to Symbolic Imagination: The Latin American Novel From 1920 to 1960." Hispania, 54, 3 (Sept., 1971), 445-451.

84. Magaña Esquivel, Antonio. Medio siglo de teatro mexicano (19001961). México: Instituto Naciona Ide Bellas Artes, 1964 [Rulfo, p. 163].

85. Martínez, José Luis. Literatura mexicana siglo XX. 1910-1949. I. México: Edicorial Porrúa (Antigua Librería Robredo), 1949 [Rulfo, p. 85].

86. _- "Unidad y diversidad", pp. 73-92. En América Latina en su literatura, César Fernández Moreno, coordinador. México: Siglo Veintiuno Editores (Serie América Latina en su cultura), 1972 [Rulfo, p. 90].

87. Mazzei, Angel. Sobre El arie de Iuan Rulfo de Rodríguez Alcalá. El Nacional (5 de junio de 1966).

88. Mead, Robert G., Jr. "El ambiente literario mexicano en 1956", pp. 76-85. En su Temas bispanoamericanos. México: Ediciones de Andrea (Colección Studium, no. 26), 1959.

89. - - "- "Actualidad y destino de la literatura iberoamericana en los Estados Unidos." La Gaceta (Fondo de Cultura Económica), $\mathrm{X}, 105$ (mayo de 1963), 5.

90. Menton, Seymour. "La estructura épica de Los de abajo y un prólogo especulativo." Hispanic, 50, 4 (Dec., 1967), 1001-1011 [Rul[o, pp. 1002-1003]. 
91. Merrell, Floyd Fenly. "Sacred-Secular Complementary in Al filo del agua and Pedro Páramo: An Inquiry into Myth Making." Albuquerque, New Mexico: The University of New Mexico, 1973. Tesis doctoral.

92. - "Sacred-Secular Complementary in Al filo del agua and Pedro Páramo: An Inquiry into Myth Making." Dissertation $A b$ stracts International, 34 (1973), 2640 A-2641 A.

93. Miliani, Domingo. "La novela mexicana de hoy." Imagen (Caracas), Suplemento no. 31 (15-31 de agosto de 1968).

94. Millán, María del Carmen. "Panorama de la literatura mexicana", pp. xi-xxvii. En Diccionario de escritores mexicanos, de A. M. Ocampo de Gómez y E. Prado Velázquez. México: Universidad Nacional Autónoma, Centro de Estudios Literarios, 1967 [Rulfo, p. xxv].

95. - Literatura mexicana (con notas de literatura y antología). De acuerdo con los programas oficiales. México: Ediciones Esfinge, 1962 [Rulfo, pp. 277, 278].

96. Molina-Foix, Vicente (con Federico Campbell). "Vicente MolinaFoix o el tema de las alternativas", pp. 52-71. En Infame turba, de Federico Campbell. Barcelona: Editorial Lumen, 1971 [Rulfo, p. 60].

97. Murray, Michele. "From South of the Border, A Florescence of Creativity." The National Observer (24 March, 1973), 25.

98. Nielsen, Delia López de. (Sobre El arte de Juan Rulfo de Rodriguez Alcalá). Vida Universitaria Monterrey (México), (20 de marzo de 1966), 8-9.

99. Oberhelman, Harley D. "The Absurd in Three Representative Spanish American Novelists", pp. 95-110. En From Surredlism to the Absurd. Proceedings of the Comparative Literature Symposium, Vol. III, Wolodymyr 'T. Zhyla, ed. Lubbock, Texas: Interdepartmental Committee on Comparative Literature, Texas Technological University, 1970 [véase Ramírez, No. 319].

100. O'Neill, Samuel Joseph, Jr. "Psychological-Literary Techniques in Representative Contemporary Novels of Mexico." Dissertation Abstracts, 27 (1966), 213-A [véase Ramírez, No. 321].

101. Oviedo, José Miguel. "Una discusión permanente", pp. 425-440. En América Latina en su literatura, César Fernández Moreno, coordinador. México: Siglo Veintiuno Editores (Serie América Latina en su cultura), 1972. 
102. Pacheco, José Emilio. "Simpatías y diferencias." Universidad de México, XVI, 12 (agosto de 1964), 30.

103. Pasamanik, Luisa. "Rulfo y la fuetza telúrica." La Gaceta (Fondo de Cultura Económica), 17 (enero de 1970), 17-19.

104. Percas Ponseti, Helena. "El arte de Juan Rulfo por Hugo Rodríguez Alcalá." Revista Iberoamericana, XXXIII, 63 (enero-junio de 1967), 169.

105. Piglia, Ricardo. "América Latina: de la traición a la conquista", pp. 7-12. En su Crónicas de Latinoamérica. Buenos Aires: Editorial Jorge Alvarez, 1968 [también, nota sobre Rulfo, p. 14; véase Ramírez, No. 74].

106. Popeanga, Eugenia. "Romanul Latino-american contemporan." Revista de Istorie Si Teorie Literara (Bucarest), XXI, 2 (1972), 347-353.

107. Quebleen, Julieta $H$. "Panorama de la novela mexicana." Universidad (Santa Fe, Argentina), no. 50 (oct.-dic. de 1961), 171-185.

108. Rabell, Malkah. "Nuevas voces en las letras mexicanas. De Juan Rulfo a Carlos Fuentes." La Nación (24 de dic. de 1960).

109. Ramirez, Arthur. "Style and Technique in Juan Rulfo." Dissertation Abstracts International, 34 (1973), 2649-A [véase Ramírez, No. 515].

110. Rama, Angel. Diez problemas para el novelista latinoamericano. Caracas: Síntesis Dosmil (Colección Manos Libres), 1972 [Rulfo, p. 47; véase Ramírez, Nos. 330, 331].

111. Rodríguez-Monegal, Emir. "Una escritura revolucionaria." Revista Iberoamericana, XXXVII, 76-77 (julio-dic. de 1971), 467-506.

112. - - "The New Latin American Novelists", pp. 9-28. En The TriQuarterly Antbology of Contemporary Latin American Literature, José Donoso and William A. Henkin, eds. New York: E. P. Dutton and Co., 1969 [Rulfo, pp. 18-19; véase Ramírez, No. 344].

113. - - "La nueva novela latinoamericana", pp. 47-63. En Actas del Tercer Congreso Internacional de Hispanistas, publicadas bajo la dirección de Carlos H. Magis. México: El Colegio de México, por la Asociación Internacional de Hispanistas, 1970 [Rulfo, pp. 53, 55; véase Ramírez, Nos. 341, 342].

114. ——. "Tradición y renovación", pp. 139-166. En América Latina en su Literatura, César Fernández Moreno, coordinador. México: Siglo Veintiuno Editores (Serie América Latina en su cultura), 1972 [Rulfo, pp. 146, 157, 158, 161].

115. Rubín, Ramón (con Emmanuel Carballo), pp. 339-357. En Dieci- 
nueve protagonistas de la literatura mexicana del siglo veinte. México: Empresa Editoriales, 1965 [Rulfo, p. 349].

116. Sarduy, Severo. "El barroco y el neobarroco", pp. 167-184. En América Latina en su literatura, César Fernández Moreno, coordinador. México: Siglo Veintiuno Editores (Serie América Latina en su cultura), 1972 [Rulfo, p. 177].

117. Shaw, Donald L. "Eduardo Mallea y la novela hispano-americana contemporánea." Razón y Fábula, no. 6 (1969), 19-24.

118. Silva Cáceres, Raúl. "Prólogo: aparición y sentido de la novela actual", pp. 9-19. En La novela bispanoamericana actual. Compilación de ensayos críticos, Angel Flores y Raúl Silva Cáceres, eds. New York: Las Américas Publishing Co., 1971 [Rulfo, p. 17].

119. Solana, Rafael. "Arreolismo y Rulfismo." La Gaceta (Fondo de Cultura Económica), 16 (junio de 1969), 2 y 3.

120. Sommers, Joseph. "The Present Moment in the Mexican Novel." Books Abroad, 40 (Summer, 1966), 261-266.

121. - "The Recent Mexican Novel: Tradition and Innovation." Inter-American Review of Bibliograpby/Revista Interamericana de Bibliografía, 16, no. 4 (1966), 398-402.

122. - - "Temas y perspectivas de investigaciones en la novela mexicana." Nueva Narrativa Hispanoamericana, III, 2 (sept. de 1973), 286-295.

123. Sueiro, Daniel (con Fernando Tola de Habich y Patricia Grieve). "Entrevista con Daniel Sueiro." Sin Nombre (San Juan, P. R.), I, 3 (ene.-mar. de 1971), 39-47.

124. 'Tovar Llorente, Antonio. Novela española e bispanocmericana. Madrid: Ediciones Alfaguara (Serie Hombre, Hechos e Ideas, XXIV), 1972 [Rulfo, p. 214 ].

125. Valadez, Edmundo. "Autores y libros." México en la Cultura (de Novedades), no. 321 (15 de mayo de 1955), 2.

126. Valdés, Mario. "The Literary Social Symbol for an Interrelated Study of Mexico." Joumal of Inter-American Studies, III (1965), 385-399.

127. Valenzuela Rodarte, Alberto. Contemporary Latin American Writers. New York: Las Américas Publishing, Co., 1971.

128. - Historia de la literatura en México e Hispanoamérica. México: Editorial Jus, 1967.

129. Valencia Goelkel, Hernando. "La mayoria de edad", pp. 121-135. En América Latina en su literatura, César Fernández Moreno, 
coordinador. México: Siglo Veintiuno Editores (Serie América Latina en su cultura), 1972 [Rulfo, p. 128].

130. Vázquez Amaral, José. The Contemporary Latin American Narrative. New York: Las Américas Publishing Co., 1970 [Rulfo, pp. 154-155].

131. Verdevoye, Paul. "Socionarrativa hispanoamericana", pp. 893-899. En Actas del tercer Congreso Internacional de Hispanistas, bajo la dirección de Carlos H. Magis. México: El Colegio de México, por la Asociación Internacional de Hispanistas, 1970 [Rulfo, p. 8951 .

132. Viñas, David (con Mario Szichman). "Entrevista." Hispaménca, I, 1 (julio de 1972), 61-67 [Rulfo, p. 66].

133. Xirau, Ramón. "Crisis del realismo", pp. 167-184. En América Latina en su literatura, César Fernández Moreno, coordinador. México: Siglo Veintiuno Editores, 1972 [Rulfo, pp. 189, 197, 199-200].

134. Yáñez, Agustín (con Emmanuel Carballo), pp. 281-324. En Diecinueve protagonistas de la literatura mexicana del siglo veinte. Mé. xico: Empresas Editoriales, 1965.

135. Zuleta Alvarez, Enrique. "2. Hispanoamérica", pp. 95-133. En Las literaturas contemporáneas en el mundo. Barcelona: Editorial Vi. cens-Vives, 1967 [Rulfo, 126-127].

VII. Reseñas y artículos sobre El llano en llamas o sus cuentos:

136. Brower, Gary. "Diles que no me maten: aproximación a su estructura y significado." Nueva Narrativa Hispanoamericana, III, 2 (sept. de 1973), 231-235.

137. Burton, Julianne. "A Drop of Rain in the Desert: Something and Nothingness in Juan Rulfo's 'Nos han dado la tierra." "Latin American Literary Review, II, 3 (Fall-Winter, 1973), 55-62.

138. Fernández, Sergio. "Advertencia", pp. 5-13, y "El mundo paralítico de Juan Rulfo", pp. 113-141. En su Cinco escritores hispanoamericanos. México: Facultad de Filosofía y Letras, Universidad Nacional Autónoma (Ediciones Filosofía y Letras, No. 30), 1958 [véase Ramírez, Nos. 244, 399].

139. [Gascón, Elvira?]. "El llano en llamas, por Juan Rulfo (Cuentos). La Creación Literaria", p. 506. En Cátálogo General 1964. México: Fondo de Cultura Económica, 1964 [nota también en la p. 388 ]. 
140. Iyon, Ted. "Ontological Motifs in the Short Stories of Juan Rulfo." Journal of Spanish Studies: Twentieth Century, I, 3 (Winter, 1973), 161-168.

141. Serno-Maytorena, M. A. "El hombre y el paisaje del campo jalisciense en 'La cuesta de las comadres', cuento de Juan Rulfo." Cuadernos Americanos, 177 (mayo-junio de 1971), 208-216.

VIII. Reseñas y artículos sobre Pedro Páramo:

142. Anon. "Nuestras recientes ediciones. Colección Popular. Juan Rulfo. Pedro Páramo." Lat Gaceta (Fondo de Cultura Económica), $\mathrm{X}, 110$ (oct. de 1963), 8.

143. Bejarano, Marino. "Comala o la irrealidad transmutada." Revista Nacional de Cultura, XXXII, nos. 209-211 (agosto-sept. de 1972), 19-25.

144. Cortés, Norman. (Sobre Pedro Páramo). Anales de la Universilad de Cbile, CXII, 131 (1964), 238-239.

145. Cuéllar, José María "Pedro Páramo (Juan Rulfo)." La Pájun a Pinta (El Salvador), II, 16 (abril de 1967), 7.

146. Luquín, Eduardo. "La novelística mexicana y una novela." Revista Mexicana de Cultura (de El Nacional), 2a. serie, no. 530 (26 de mayo de 1957), 6 [véase Ramírez, No. 305].

147. Martínez, Ricardo. "Rulfo. J.: Pedro Páramo (Novela)", p. 506. En Catáloga general 1964. México: Fondo de Cultura Económica, 1964.

148. Pupo-Walker, Enrique. "La creación de personajes en Pedro Páramo: notas sobre una tradición." Anali Istituto Universitario Orientale, Sezione Romanza (Napoli), XIV, (Gennaio, 1972), 97-105.

149. Rodríguez-Luis, Julio. "Algunas observaciones sobre el simbolismo de la relación entre Susana San Juan y Pedro Páramo." Cuadernos Hispanoamericanos, 270 (dic. de 1972), 584-594.

150. Rodríguez Monegal, Emir. "Relectura de Pedro Páramo." Papel Literario (de El Nacional, Caracas), (26 de oct. de 1969), sin pp.

151. Serra, Edelweis. "Estructura de Pedro Piramo." Nueva Narrativa Hispanoamericana, III, 2 (sept. de 1973), 211-229.

152. Van Praag, J. A. "Juan Rulfo: Pedro Páramo." Norte, III, 1 (sept. de 1962), sin pp. [véase Ramírez, No. 491]. 
